\section{How I manage frontline transplant-ineligible multiple myeloma}

\author{
Daniele Derudas, ${ }^{1}$ Francesca Capraro, ${ }^{1}$ \\ Giovanni Martinelli, ${ }^{2}$ \\ Claudio Cerchione ${ }^{2}$ \\ ${ }^{1}$ S.C. di Ematologia e C.T.M.O., \\ Ospedale Oncologico di Riferimento \\ Regionale "A. Businco", Cagliari; \\ ${ }^{2}$ Hematology Unit, Istituto Scientifico \\ Romagnolo per lo Studio e la Cura dei \\ Tumori (IRST) IRCCS, Meldola (FC), \\ Italy
}

\begin{abstract}
The Multiple Myeloma (MM) is a plasma cells hematological malignancy with a median age of 69 years at diagnosis. The autologous stem cell transplantation is the standard of care for this disease but less than half of newly diagnosed patients are assessed for this treatment due to comorbidities or complications of disease. The management of transplant ineligible MM patients is based on the balance safety and efficacy of the new available regimen and a careful assessment of the frailty status is mandatory to define the goals. In this review we discuss of the clinical dilemmas in the management and define how to manage them based on the evidence from clinical trials and "real life" experience.
\end{abstract}

\section{Introduction}

Multiple Myeloma (MM) is an hematological malignancy characterized by an abnormal proliferation of monoclonal plasma cells. It accounts for $10 \%$ of all the hematological neoplasms.

The diagnosis requires the documentation of $\geq 10 \%$ monoclonal plasma cells and one or more markers of active disease defined with the acronym CRAB $(\mathrm{C}=$ elevated serum calcium, $\mathrm{R}=$ renal impairment, $\mathrm{A}=$ anemia, $\mathrm{B}=$ lytic bone lesions). ${ }^{1}$ Recently new biomarkers of malignancy have been introduced as myeloma defining events (MDEs) in absence of CRAB features. These MDEs are represented by bone marrow clonal plasma cells $\geq 60 \%$, an involved/uninvolved serum free light chains ratio $\geq 100$ and/or presence of $>1$ lytic lesions on magnetic resonance imaging (MRI). ${ }^{2}$

The median age at diagnosis of MM is
69 years, approximately $70 \%$ are older than 65 years and $40 \%$ are older than 75 years. ${ }^{3-5}$ Moreover it is predictable the in 2050434 million people in the world will be older than age $80^{6}$ with 150000 / year newly diagnosed MM over 80 years.

In the last decades it has been observed a significant improvement either in the Progression Free Survival (PFS) and Overall Survival (OS) of the MM patients associated with the introduction of novel agents (immunomodulatory drugs, proteasome inhibitors, monoclonal antibodies) and mainly the more extensive use of highdose therapy followed by autologous hematopoietic stem cell transplantation (aHSCT) and maintenance treatment. ${ }^{7-9}$

However several studies have shown a more marginal benefit in the elderly population (particularly over 75 years and older). That could be explained by an higher rate of comorbidities and organ dysfunction associated with the aging in this setting of MM patients, that exclude the most of this population from aHSCT. ${ }^{7-9}$

Either a close evaluation of the frailty with a geriatric assessment either a management of comorbidities is mandatory to exploit the efficacy of the association of the new agents and immunotherapy drug and to reduce the risk of treatment related toxicities with an improvement of the treatment efficacy in terms of PFS/OS and quality of life (QoL).

The management of elderly MM patients requires as first step the evaluation of transplant eligibility and then the assessment of frailty in order to define the best therapy available.

\section{Evaluation of transplant-eligibili- ty and frailty score}

Historically the age cut-off accepted in clinical trials for eligibility for aHSCT was 65 years. But this limit excluded more than two-third of newly diagnosed MM patients considering the median age at diagnosis. In the clinical practice the age cut-off is extended to $70-75$ years..$^{10,11}$

The European Society of Medical Oncology (ESMO) recommended aHSCT up to the age of 70 years, contrariwise, the National Comprehensive Cancer Network (NCCN) did not set an age cut-off. ${ }^{12,13}$

Recently it has been documented an increasing use of aHSCT in the older population considering mostly retrospective or population based studies. A first analysis evaluated the trend of aHSCT in 31 European countries with a rate of aHSCT in patients older than 65 years of $3 \%$ between
Correspondence: Daniele Derudas, S.C. di Ematologia e C.T.M.O., Ospedale Oncologico di Riferimento Regionale "A. Businco", via Jenner snc, 09121 Cagliari (CA), Italy.

E-mail: daniele.derudas@aob.it

Claudio Cerchione, Hematology Unit, Istituto Scientifico Romagnolo per lo Studio e la Cura dei Tumori (IRST) IRCCS, Via P. Maroncelli 40, 47014, Meldola (FC), Italy.

E-mail: claudio.cerchione@irst.emr.it

Key words: Multiple Myeloma; Stem cell transplantation; Frailty.

Conflict of interest: The authors declare no potential conflict of interest

This work is licensed under a Creative Commons Attribution-NonCommercial 4.0 International License (CC BY-NC 4.0).

${ }^{\circ}$ Copyright: the Author(s), 2020

Licensee PAGEPress, Italy

Hematology Reports 2020; 12(s1):8956

doi:10.4081/hr.2020.8956

1991 and 1995 versus $18 \%$ between 2006 and 2010. Costa et al. described similar data in USA and Canada. ${ }^{14,15}$

Subsequent trials demonstrated the feasibility of aHSCT in patients aged $>65$. The most prolonged hospitalization and most frequent post- aHSCT complications observed in several clinical trials did not translate in higher treatment-related mortality (TRM) compared to younger patients, probably due to a reduction of conditioning regimen (from 200 to $100-140 \mathrm{mg} / \mathrm{m}^{2}$ of melphalan). Data from trial including tandem transplant followed by consolidation and maintenance showed an increased TRM $(19 \%)$ in patients older than 70 years compared with younger patients. ${ }^{16-21}$

These data encourage the use of aHSCT in older patients but only after a careful selection of patients, that must be based on the evaluation of the fitness with geriatric assessment (GA) tool, consideration of alternative therapies and the availability of caregiver collaboration, in order to reduce the rate of complication and TRM. In absence of clear data from clinical trials the optimal dose of melphalan has not been established. However in patients $>70$ years, with renal impairment, and/or performance status less than $90 \%$ is reasonable to reduce the dose of melphalan to $100-140 \mathrm{mg} / \mathrm{m}^{2}$.

Considering the efficacy of new combination with second-generation proteasome inhibitors, immunomodulatory agents and monoclonal antibodies the role of aHSCT in older patients is challenging. For example, recently the MAYA and ALCYONE trials including the addition of Daratumumab 
with standard regimes in transplant-ineligible population (respectively Daratumumab plus Lenalidomide-Dexamethasone DaraRd - and Daratumumab plus Bortezomib-Melphalan-Prednisone DaraVMP) showed a significant decreasing of risk of death or progression (HR 0,56 and $0,43)$ and high rates of minimal residual disease (MRD) negativity $(25 \%) .^{22,23}$

Once defined the ineligibility for ASCT, the choice of the best regimen depends on the fitness of the patients. In fact the elderly population is heterogeneous and the aging is associated with a high rates of comorbidities and diseases that could exacerbate the side-effects of the available drug combination and increase the morbidity and mortality. For example, uncontrolled diabetes could lead to medical complication due to steroids treatment and increase myelomarelated mortality. 24

Pre-existent peripheral neuropathy may limit the therapy with thalidomide and bortezomib. Cardiovascular diseases (cardiac arrhythmias, congestive cardiac failure, uncontrolled arterial hypertension) need a careful assessment in case of treatment with proteasome inhibitors (PI) particularly carfilzomib. Immunomodulatory drugs (IMIDs), as lenalidomide and thalidomide, could not be an optimal option in case of previous history of thrombosis. Renal failure affected a high proportion of patients with diagnosis of MM and could need an adjustment of the dosage of some drugs like lenalidomide.

The comorbidities, the decreased organ function and the frailty make the patients more vulnerable to the side effects of therapy with a reduced adherence to therapy, early discontinuation and decreased treatment efficacy. Bringhen et al. have showed in a large meta-analysis of 1435 elderly patients enrolled in 4 randomized trials treated with thalidomide and/or bortezomib, that the risk of death was higher in patients aged $\geq 75$ years (HR $1,44,95 \%$, CI 1,20 $1,72, p<0,01)$, in patients affected by renal failure (HR 2,02, 95\%, CI 1,51 - 2,70, $p<$ $0,01)$ and in those that developed grade 3-4 infections and/or cardiac and gastrointestinal events during the treatment (HR 2,53, $95 \%$, CI $1,74-3,64, p<0,01)$. Also the patients that needed a discontinuation due to adverse events (HR 1,67, 95\%, CI 1,12 $2,51, p=0,01)$ showed a worse increased risk of death. The estimated 3-years OS was $68 \%$ in patients $£ 75$ years and $57 \%$ in those 375 years (HR 1,44, 95\%, CI 1,2 - 1,72, $p<$ $0,01)^{25}$

A frailty status may affect significantly the compliance to therapy: for example the management of oral therapy may be difficult in presence of mental or cognitive impairments with requirement of caregiver and, on the other hand, an intravenous or subcutaneous treatment, with the need of frequent hospital accesses, could be a limitation for patients with mobility impairment or in absence of caregiver. For these reasons recently different morbidity scores and GA tools were introduced and implemented to evaluate the fitness of patients and consequently define the goal of therapy with the best option available according to the frailty status by preventing and managing the side effects of chemotherapy and limiting the discontinuation of the treatment

In fact the terms aging and frailty represent different concepts. The aging is associated with a physiological reserve decline while the frailty is a complex syndrome characterized by an increased vulnerability and physiological decline. For this reason, because of heterogenicity of chronological and functional age, it is needed a multidimensional assessment that consider the age, comorbidities, performance status, nutritional status, polypharmacy, cognition, socioeconomics factors to define the frailty of transplant- ineligible MM patients.

The International Myeloma Working Group (IMWG) frailty index categorizes MM patients as fit (score 0, 39\%), unfit (score $1,31 \%$ ) and frail $(\geq 2,30 \%)$ ) using as domains age, comorbidities (Charlson Comorbidity Index) and functional status of patients (Katz Activities of Daily Living, ADL and Lawton's Instrumental Activities of Daily Living, IADL). ${ }^{26}$ This tool has not been validated in "real life" but only in clinical trial ${ }^{27}$ and the patient aged $>80$ years are defined as frail by definition. The IMWG frail score defines a worse PFS and OS with increased discontinuation of therapy for the frail category. The fit group shown an 3-years OS of $84 \%$, the intermediate fit $76 \%$ and frail population $57 \%$ (HR 3,57: $p<0,01)$. The frail patients documented also a higher risk of grade $3 / 4$ non hematological toxicities compared with the other groups (HR 1,57; 95\%; CI 1,12 - 2.2). This score can be calculated with web application http://www.myelomafrailtyscorecalcutator.net/.

Another tool, the revised myeloma comorbidity index (R-MCI), categorizes the patients according to Karnofsky Performance Status (KPS), frailty, age, lung and renal function and cytogenetics. It was set in Germany on a cohort of 801 newly diagnosed MM (NDMM) consecutive patients. The categorization is between fit (Index $\leq 3$ ), intermediate (Index 4-6), frail patients (Index $>6$ ) with a median OS of $10,1,4,4$ and 1,2 years respectively. ${ }^{28}$

Regarding other fitness assessment, the Cancer and Aging Research Group
Geriatric assessment tool has been used to define the ASCT eligibility and the Healthrelated Quality of Life assessment represents an important tool in the clinical trials to define complementary endpoints particularly in transplant-ineligible MM patients.

Unfortunately there is not an accordance between the different GA tools: for example a comparison of IMWG frailty score with Freiburg Comorbidity Index (based on renal function, KPS and Lung impairment) shown a discordance of $57 \%$ in patients categorization. ${ }^{29}$

The frailty assessment, regardless the tool available, is, in conclusion, mandatory in this setting of patients to tailor and optimize treatment both in clinical trial and in the real-life practice.

In our Department we select the fit patients for aHSCT between $65-70$ years by IMWG frailty assessment and availability caregiver support. The patients non eligible to HSCT are stratified by IMWG frailty assessment upfront and during relapse.

\section{The treatment of newly diag- nosed transplant-ineligible MM patients}

Once defined the ASCT ineligibility and the fitness status, the goal of treatment must lead the choice of the best regimen (Table 1).

In the last decades the introduction of novel agents has expanded dramatically the availability of effective regimen. The backbone of the treatment in this setting was the alkylator-based therapy, typically oral melphalan-prednisone (MP). The first novel agent associated with MP was thalidomide (MPT). Seven randomized clinical trials tested this regimen ${ }^{30-36}$ in transplant ineligible population: all the trials demonstrated an improvement in PFS but only four out of seven showed a benefit in order of OS. However the introduction of thalidomide was associated with a increased risk of deep venous thrombosis, peripheral neuropathy, constipation and high rates of treatment discontinuation and dose reduction. For these reasons this regimen is currently replaced by other effective and less toxic treatments.

Another association of new drugs with MP is the triplet bortezomib - melphalan prednisone (VMP). The VISTA trial ${ }^{37,38}$ showed superiority versus MP in terms of PFS and OS (median 24 vs 16,6 months, HR $0,48 \mathrm{p}<.001$ and median 56,4 vs 43,1 months, HR $0,695 \mathrm{p}<.001$ respectively) and these results was confirmed in different sub-groups by age ( $\geq 75$ years), disease stage (ISS stage III) and renal function 
(eGFR $<60 \mathrm{ml} / \mathrm{min}$ ). Because of the high rates of peripheral neuropathy associated with Bortezomib it was introduced a oneweekly and sub-cutaneous dosing with reduction of the side effects incidence without difference in efficacy. ${ }^{39,40}$

In the GIMEMA-MM-03-05 trial the regimen VMP-thalidomide followed by bortezomib-thalidomide (VT) maintenance (VMP-VT) for 2 years was compared with VMP $^{41,42}$ The median PFS after a follow-up of 54 months and the 5 -years OS was superior with VMP-VT regimen.

An alkylator-free regimen was evaluated by the PETHEMA group with the comparison of bortezomib-thalidomide- prednisone (VTP) versus VMP. ${ }^{43,44}$ After induction the patients were randomized between VT and bortezomib-prednisone (VP). The trial showed a median PFS of 32 months for VMP and 23 months for VTP $(p=.09)$ with a prolonged OS in the VMP arm (median 63 vs 43 months; HR:0,67, $\mathrm{p}=.01$ ). No difference was observed in OS between the two maintenance arms with PFS pf 32 months for VT vs 24 months of VP. The VT regimen was affected by a higher rate of cardiovascular adverse events. Most recently, considering the high rates of side effects associated with thalidomide, different trials tested preferably lenalidomide in combination for the treatment of transplant ineligible MM patients.

The association of lenalidomide with MP (MPR) was evaluated in two trial,
ECOCG E1A06 and MM-015, ${ }^{45,46}$ the first in comparison with MPT and the last versus MP and MPR-R (maintenance with continuous lenalidomide). The trials fail to demonstrate a superiority of MPR to MPT or MP in terms of PFS and PS. The MM015 study shown only a PFS advantage in the MPR-R arm versus MPR because of the maintenance with Lenalidomide. Notably it was found high rates of grade III/IV hematological adverse events in the MPR arm, particularly for older patients ( $\geq 75$ years).

The unacceptably hematological toxicity of the association of lenalidomide with melphalan shows that lenalidomide is not an optimal partner with alkylating agents highlighting the need of different combination.

The FIRST trial is a three arms trial that compared fixed therapy with Rd (18 cycles, Rd18) versus MPT and continuous Rd. This study demonstrated the superiority of continuous Rd combination versus MPT and Rd18 in terms of reduction of risk of death (HR 0,72, p =.0006 and HR 0,70, p=.0001) without difference in median PFS between MPT and Rd18. These advantages were observed in different subgroups (age, ISS, renal function, performance status) but not in the high risk population (defined for high-risk cytogenetics or elevated LDH). No difference in OS was observed between continuous $\mathrm{Rd}$ and $\mathrm{Rd} 18$ but Rd increased OS of patients compared with MPT. Notably a quality of life assessment was performed with a better result in the group of continuous Rd versus MPT, also because a low rate of side effects. ${ }^{47,48}$

The association of lenalidomide with bortezomib and dexamethasone (VRd) in comparison with $\mathrm{Rd}$ was explored in the SWOG S0777 trial that enrolled newly diagnosed MM patients without an intent of immediate ASCT. A twice-weekly intravenous bortezomib schedule was defined for this trial. The study showed an improved PFS (43 vs 30 months, HR 0,712; p = 0.0038) and OS (75 vs 64 months; HR $0.709 ; 0.025)$ in the VRd arm if compared with $\mathrm{Rd}$ regimen. The advantages remain after age-adjusted multivariate analysis. Evaluation of the population $>75$ years old shown a better PFS and OS in the VRd arm. As expected the rate of grade $3 / 4$ peripheral neuropathy was superior in the VRD arm (33\% vs $11 \%$ ) and grade $3 / 4$ toxicities were described in $82 \%$ of VRd arm versus $75 \%$ in $\mathrm{Rd}$ arm. ${ }^{49}$ Despite the encouraging results no clear conclusions can be made because the trial was not restricted to transplant ineligible patients.

In a Phase II dose-reduced association of lenalidomide, bortezomib and dexamethasone (VRd-lite), lenalidomide was administered as a single day dose of $15 \mathrm{mg}$ (days 1-21), bortezomib subcutaneous at a dose of $1,3 \mathrm{mg} / \mathrm{m}^{2}$ once-weekly (days $1-8$ 1-5-22) and dexamethasone at dose of 20 $\mathrm{mg}$ (days 1,2-8,9-15,16 and 22,23) if $<75$ years old or on days $1-8-15-22$ for patients

Table 1. Selected regimens in newly diagnosed transplant-ineligible multiple myeloma.

\begin{tabular}{|c|c|c|c|c|c|c|}
\hline Study & Regimen & $\begin{array}{l}\text { Median age in } \\
\text { years (range) }\end{array}$ & ORR (\%) & $\begin{array}{c}\text { Grade } 3 \text { AE rates } \\
(\%)\end{array}$ & $\begin{array}{l}\text { Median PFS } \\
\text { (months) }\end{array}$ & $\begin{array}{l}\text { Median OS } \\
\text { (months) }\end{array}$ \\
\hline Fayers et al. & $\begin{array}{l}\text { MPT us MP meta- } \\
\text { analysis of six } \\
\text { randomized trials } \\
n=1685)\end{array}$ & $72-78,5 y$ & NR & NR & $\begin{array}{c}20,3 \text { vs } 14,9 \\
(\operatorname{HR} 0,68: p<0,001)\end{array}$ & $\begin{array}{c}39,3 \text { vs } 32,7 \\
\text { (HR0,83; } p=0,004)\end{array}$ \\
\hline San Miguel et al. & $\begin{array}{c}\text { VMP } \\
\text { vs } \mathrm{MP}(\mathrm{n}=682)\end{array}$ & $\begin{array}{c}71(57-90) \text { vs } 71 \\
(48-91) \text { y }\end{array}$ & $\geq P R 71$ vs 35 & 81 us 71 & $\begin{array}{c}\text { mTTP: } 20,7 \text { vs } 15 \\
\text { (HR 0,54; } p<0,001)\end{array}$ & $\begin{array}{c}56 \text { vs } 43 \\
(\operatorname{HRR} 0,7 ; p<0,001)\end{array}$ \\
\hline Benbouker et al. & $\begin{array}{c}\text { cRd vs } \mathrm{Rd} 18 \\
\text { vs MPT ( } \mathrm{n}=1623)\end{array}$ & $\begin{array}{c}73(44-91) \text { vs } 73(40-89) \\
\text { vs } 73(51-92) \mathrm{y}\end{array}$ & 75 vs 73 us 62 & 85 us 80 us 89 & $\begin{array}{c}25,5 \text { us } 20,7 \text { (HR } 0,7 ; \\
p<0,001) \text { vs } 21,2 \\
(\operatorname{HR} 0,72 ; p>0,001)\end{array}$ & $\begin{array}{c}4 \text { years OS: } 59 \% \text { us } \\
56 \% \text { (HR 0,9; } p=0,31 \text { ) } \\
\text { us } 51 \% \text { (HR 0,78; } \\
p=0,002 \text { compared to cRd) }\end{array}$ \\
\hline Durie at al. & $\begin{array}{c}\text { VRd us Rd us } 6 \\
\text { Maintenance } \mathrm{Rd} \\
\text { in both arms }(\mathrm{n}=525)\end{array}$ & $63(56-71)$ y & 82 vs 72 & 82 vs 75 & 43 vs 30 (HR 0,71; $p=0,002)$ & 75 us 64 (HR 0,709; $p=0,025)$ \\
\hline Mateos et al. & $\begin{array}{c}\text { D-VMP us VMP } \\
(\mathrm{n}=700)\end{array}$ & $\begin{array}{c}71(40-93) \\
\text { vs } 71(50-91) \text { y }\end{array}$ & 91 vs 74 & 41,6 vs 32,5 & $\begin{array}{l}\text { 18-months PFS: } 72 \text { us 50\% } \\
\text { (HR } 0,5 ; p<0,001)\end{array}$ & OS data immature \\
\hline Facon et al. & DRd us Rd ( $\mathrm{n}=737)$ & $73(45-90)$ y & VGPR: 79 us 53 & NR & NR us 31,9 (HR 0,$55 ; p<0,0001)$ & OS data immature \\
\hline Palumbo et al. & $\begin{array}{c}\text { MPRx9 f/b R } \\
\text { maintenance vs } \\
\text { MPRx9 us MPx9 } \\
(\mathrm{n}=459)\end{array}$ & $\begin{array}{c}71(65-87) \text { us } 71(65-86) \\
\text { vs } 72(65-91) \mathrm{y}\end{array}$ & 77 vs 68 vs 50 & $\begin{array}{c}\text { Grade } 4 \text { neutropenia/ } \\
\text { thrombocytopenia: } \\
3 \% / 11 \% \text { us } 32 \% / 12 \% \text { us } \\
12 \% / 4 \%\end{array}$ & $\begin{array}{c}31 \text { vs } 14 \text { (HR } 0,49 ; \\
p<0,001) \text { us } 13 \text { (HR } 0,4 ; \\
p<0,001 \text { compared to MPR-R) }\end{array}$ & $\begin{array}{c}\text { 3-years OS: } 70 \% \text { vs } \\
62 \% \text { (HR 0,79; } p=0,25) \\
\text { us } 66 \% \text { (HR 0,95; } \\
p=0,81 \text { compared to MPR-R) }\end{array}$ \\
\hline
\end{tabular}

cRd: continuous lenalidomide-dexamethasone; HR: hazard ratio; D-VMP: daratumumab-bortezomib-dexamethasone; DRd. daratumumab-lenalidomide-dexamethasone; MP: melphalan-prednisone; MPT; melphalanprednisone-thalidomide; MPR: melphalan-prednisone-lenalidomide; NR: not reported; ORR: overall response rate (partial response or better); OS: overall survival ; PFS: progression free survival; PR: partial response; $\mathrm{R}$ = lenalidomide; Rd: lenalidomide-dexamethasone; Rd18: lenalidomide-dexamethasone for 18 cycles; SAE: serious adverse events; TTP: time to progression; VRd: bortezomib-lenalidomide-dexamethasone; VMP: bortezomib-melphalan-prednisolone; VGPR: very good partial response 
older than 75 years. Every cycle was administered over 35 days. The median age was 73 years (range 65-91 years).

The overall response rate was $81,8 \%$ with an acceptable safety profile. ${ }^{50}$

The new proteasome inhibitor carfilzomib in association with MP was compared with VMP in the phase III CLARY$\mathrm{ON}$ trial. No statistical differences were found between the two arms in terms of PFS and OS. As expected in the VMP was described a higher rate of peripheral neuropathy and the KMP arm was characterized by an elevated incidence of cardiovascular events.

Two studies evaluated the role of daratumumab, anti-CD38 antibody, in the treatment of transplant ineligible MM patients.

The first one is the ALCYONE trial ${ }^{51}$ that compared 9 cycles of VMP with or without daratumumab (Dara-VMP). In the Dara-VMP arm daratumumab continued as maintenance until disease progression. The study was tailored for transplant-ineligible patient and showed in the Dara-VMP arm a reduced risk of progression and death $(0,5$; 95\% CI) and an increased rate of MRD negativity $\left(10^{-5}\right)$. The result in terms of PFS was confirmed in the high-risk group, but is was not statistically significant. The safety report showed an increased rate of pneumonia (grade 3/4) in the daratumumab group.

The second trial incorporating daratumumab was the MAIA trial, ${ }^{52}$ with the association of the monoclonal antibody with $\mathrm{Rd}$ (Dara-Rd) compared to Rd. Also for this trial the population considered was the transplant ineligible patients and the randomization was stratified according to age and geographic region. The treatment was continued until progression. After a median follow-up of 28,8 months the PFS was not reached in the Dara-Rd arm versus 31,9 months in the $\mathrm{Rd}$ arm. The overall response rate was superior in the daratumumab arm as well as the rate of deeper responses and MRD negativity to the level $10^{-5}$. Dara-Rd arm remains superior in all the subgroups analyzed but no conclusion was derived about the high-risk cytogenetic group due to the small number of patients.

There are no clinical trials tailored for transplant ineligible patients with high risk disease, for which the only information that it can be used for the treatment of this population derived from subgroup analysis of clinical studies. Despite the absence of clear survival benefit for the association available in the high-risk patients, the IMWG recommends combination of proteasome inhibitor with immunomodulatory agent and dexamethasone like RVd-lite in this setting. Conclusive data regarding new agents as carfilzomib and daratumumab are not available
According to the regimens in use, the frailty stratification and the personalized goal of therapy, the following treatment selection could be proposed (Figure 1):

1. fit patients should achieve a complete response and MRD negativity with an increased PFS and OS by full dose triplets or new quadruplets (Dara-VMP, Dara-Rd, VRd, VCd, VMP, Rd) if not assessed for ASCT.

2. intermediate fit patients need a reducedintensity regimens (weekly VMP, weekly VCd, Vd, Rd, Rd-R, VRd lite) to results in a good response, relieve symptoms and increase the overall response rate (ORR) and PFS.

3. the goal in the treatment of frail population is improve the quality of life and relieve the symptoms trough a lowtoxic and dose-adjusted regimens (rd, vd). Palliation and supportive care represent an option for many of this setting of patients.

Different treatment approaches are applied in Europe compared with the USA and other countries because of the difference in approval processes by European Medicines Agency (EMA) and Food and Drug Administration (FDA) as well as the heterogeneity of national health care systems.

In Europe, ${ }^{12}$ the two regimens approved by EMA are the VMP and Rd. About the other combination either MPT either MPR are available according with EMA state- ment but are not used in the clinical practice because of their high toxicity and low efficacy compared with Rd.

Notably the association bortezomib, cyclophosphamide and dexamethasone (VCD) is widely used but is not approved by EMA because of lack of confirmed data.

In the USA, ${ }^{53,54}$ the FDA approved various regimens comprised the association with daratumumab with VMP and Rd.

The preferred and most widely used regimen is VRd (8-12 cycles followed by Lenalidomide maintenance) and second option is DaraRd (adding cost and long term toxicity). The VCD is also recommended for treatment of this population because of high response rate.

Unlike European approach, melphalanbased regimens are not recommended and used in this population due the concern of the development of stem cell damage followed by secondary myelodysplastic syndromes and leukemia.

In Italy the standard regimens approved by the Italian Drugs Agency, named AIFA (Agenzia Italiana per il Farmaco) are VMP and $\mathrm{Rd}$. In the clinical practice is preferred the VMP association with subcutaneous and once-weekly bortezomib administration. A randomized trial comparing the two standards is ongoing (REAL trial). The choice, waiting for the results of the trials, is based on the patient and disease features, compliance, patient preference and logistics.

In our Department we consider that the

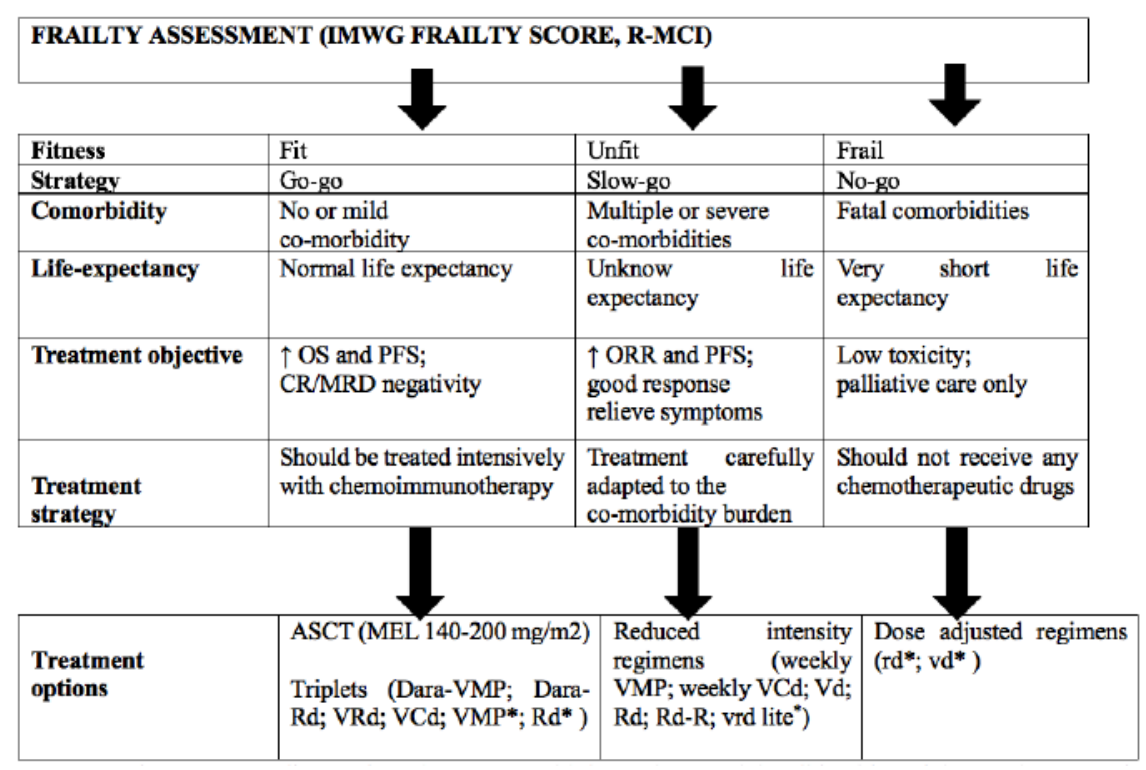

ASCT: autologous stem cell transplantation; MM, multiple myeloma; Rd, lenalidomide and dexamethasone; Rd-R, lenalidomide and dexamethasone followed by lenalidomide maintenance; R-MCI, revised myeloma comorbidity index; $\mathrm{VCd}$, bortezomib, cyclophosphamide, dexamethasone; VMP, bortezomib, melphalan, and prednisone; VRd/vrd, bortezomib, lenalidomide, and dexamethasone. $\left(^{*}\right)$ If daratumumab-based combinations or VRd are unavailable. $\left({ }^{\circ}\right)$ The lowercase letter indicates a reduced dose.

Figure 1. Management of transplant-ineligible MM patients according to frailty score 
patients with the high-risk disease may benefit from proteasome inhibitor in a weekly subcutaneous VMP regimen and in presence of renal failure bortezomib represents the gold standard. The Rd, considering the oral administration and long-term tolerability in absence of peripheral neuropathy represents an optimal option particularly in patients frail, without caregiver and living far from the Hospital. The VCD is a widely used in patients with renal failure and intermediate fit population.

\section{The treatment of relapsed/refrac- tory transplant-ineligible MM patients}

In clinical practice an asymptomatic biochemical relapse is currently managed according to the "wait and watch" strategy. ${ }^{55}$ In the other hand in case of rapid increasing (3 months) of monoclonal component or the onset of clinical sings and/or symptoms the treatment must be started. Different factors should be considered before the choice of the therapy and its aggressiveness: age, fitness status, type and duration of response to the previous therapy, cytogenetic status.

In general it is preferred a treatment with drug with non-cross reagent mechanism of action. The re-challenge could be considered if the previous therapy was associated with an interval response between 6 and 12 years, low toxicities and good tolerance.

Alternately a short duration of remission and suboptimal response need a different therapeutic strategy. New agents as second-generations proteasome inhibitors (carfilzomib and ixazomib), monoclonal antibodies (daratumumab and elotuzumab) and Immunomodulators agents like pomalidomide are effective in this setting of patients.

Particularly ixazomib, an novel proteasome inhibitor structurally different from Bortezomib, in association with $\mathrm{Rd}$ seems to be safe and efficacious in this population and its oral administration attractive in elderly patients. ${ }^{56-58}$ Elotuzumab, an anti-CS1 monoclonal association, is associated with an increased PFS when associated with Rd and is quite safe with a low rate of infusion reaction. 59,60

Carfilzomib administered with dexamethasone and daratumumab in association with $\mathrm{Rd}$ or Vd maintain efficacy in terms of PFS even in patients $>75$ years. ${ }^{61-64}$

According with the refractoriness of patients to Lenalidomide or Bortezomib is possible to add new agents like carfilzomib, daratumumab, elotuzumab or ixazomib to
$\mathrm{Rd}$ and daratumumab to $\mathrm{Vd}$.

Unfortunately there is a lacking of trials tailored to elderly relapsed/refractory $\mathrm{MM}$ patients for which is to date difficult to translate clinical information in the "real life" practice.

\section{Conclusions}

The majority of patients affected by MM are $>65$ years old and, despite the increased PFS and OS observed in the past 20 years, this advantage have not translated entirely in this population.

There are various medical unmet needs in the management in transplant ineligible MM patients:

The eligibility of ASCT has to be defined by strictly parameters widely recognized to spread this option also in the elderly population.

An accurate assessment of fitness and frailty status are mandatory to define the goals, plan the best treatment available and tailor the therapy. Therefore, it is needed GA tools reliable and efficacy to achieve this goal.

There is a lack of trials tailored for regimens according to frailty status

Different subgroups of this population, as frail and high-risk patients, are underrepresented in clinical trials.

Different treatment approaches and drugs availability, particularly between European countries and North-America translate in a heterogeneity in the management of this population.

According to the data from clinical trials and the application of concept of frailty, despite the limitations described above, to date the management of transplant ineligible patients is based in a tailored treatment with dose adjustment and careful supportive care. The introduction of second -generations agents are currently ongoing and new regimens will be exploited soon for these patients to achieve an improvement of survival and quality of life, similar to younger MM patients.

\section{References}

1. Kyle RA, Rajkumar SV. Criteria for diagnosis, staging, risk stratification and response assessment of multiple myeloma. Leukemia. 2009; 23 (1): 3-9,

2. Rajkumar SV, Dimopoulos MA, Palumbo A, Blade J, Merlini G, Mateos $\mathrm{M}-\mathrm{V}$ et al. International myeloma working group updated criteria for the diagnosis of multiple myeloma. Lancet Oncol. 2014; 15(12):e538-e48.
3. Howlander N, Noone AM, Krapcho M, Miller D, Bishop K, Alterkruse SF et al. SEER Cancer Statistics Review. 1975 2013. National Cancer Institute. Bethesda, MD, based on November 2015 SEER data submission, posted to the SEER web sire, April 2016. SEER Website 2015. http://seer.cancer. gov/archive/csr/1975 2013/.

4. Palumbo A, Bringhen S, Ludwig H, Dimopoulos MA, Bladè J, Mateos MV et al. Personalized therapy in multiple myeloma according to patient age and vulnerability: a report of the European Myeloma Network (EMN). Blood. 2011; 118 (17):4519-29.

5. van de Donk NW, Lokhorst HM. New developments in the management and treatment of newly diagnosed and relapsed/refractory multiple myeloma patients. Expert Opin Pharmacother 2013;14:1569-73.

6. 31 Zeegman

7. Mateos MV, San Miguel JF: How should we treat newly diagnosed multiple myeloma patients?. Hematology Am Soc Hematol Educ Program. 2013, 2013:488-95.

8. Pulte D, Gondos A, Brenner H: Improvement in survival of older adults with multiple myeloma: results of an updated period analysis of SEER data. Oncologist. 2011, 16:1600-1603.

9. Kumar SK, Rajkumar SV, Dispenzieri A, et al.: Improved survival in multiple myeloma and the impact of novel therapies. Blood. 2008, 111:2516-20.

10. Morgan GJ. Transplants for the elderly in myeloma. Blood. 2013;122:13321334.

11. Moreau P, Attal M, Facon T. Frontline therapy of multiple myeloma. Blood. 2015;125:3076-3084.

12. Moreau P, San Miguel J, Sonneveld P, et al; ESMO Guidelines Committee. Multiple myeloma: ESMO clinical practice guidelines for diagnosis, treatment and follow-up. Ann Oncol. 2017;28:iv52-iv61.

13. Kumar SK, Callander NS, Alsina M, et al. Multiple myeloma, version 3.2017: NCCN clinical practice guidelines in oncology. J Natl Compr Canc Netw. 2017; 15:230-269.

14. Costa LJ, Brill IK, Omel J, et al. Recent trends in multiple myeloma incidence and survival by age, race, and ethnicity in the United States. Blood Adv. 2017; 1:282-287.

15. Auner HW, Szydlo R, Hoek J, et al. Trends in autologous hematopoietic cell transplantation for multiple myeloma in Europe: increased use and improved outcomes in elderly patients in recent 
years. Bone Marrow Transplant. 2015;50:209-215.

16. Nadiminti K, Strouse C, Vikas P, et al.A single autologous stem cell transplant (ASCT) followed by two years of posttransplant therapy in recently diagnosed elderly multiple myeloma (MM) patients: safety and response results from the prospective phase II Trial (NCT01849783). Presented at: 60th Annual Meeting of the American Society of Hematology. San Diego, CA; 2018. Abstract 2153.

17. Merz M, Neben K, Raab MS, et al. Autologous stem cell transplantation for elderly patients with newly diagnosed multiple myeloma in the era of novel agents. Ann Oncol. 2014;25:189-195.

18. Ozaki S, Harada T, Saitoh T, et al; Japanese Society of Myeloma; European Myeloma Network. Survival of multiple myeloma patients aged 6570 years in the era of novel agents and autologous stem cell transplantation: a multicenter retrospective collaborative study of the Japanese Society of Myeloma and the European Myeloma Network. Acta Haematol. 2014;132:211-219.

19. Sanchez L, Sylvester M, Parrondo R, et al. In-hospital mortality and post-transplantation complications in elderly multiple myeloma patients undergoing autologous hematopoietic stem cell transplantation: a population-based study. Biol Blood Marrow Transplant. 2017;23:1203-1207.

20. Gay F, Magarotto V, Crippa C, et al. Bortezomib induction, reduced-intensity transplantation, and lenalidomide consolidation-maintenance for myeloma: updated results. Blood. 2013;122:1376-1383.

21. Dimopoulos MA, Mateos M-V, Cavo $\mathrm{M}$, et al. One-year update of a phase 3 randomized study of daratumumab plus bortezomib, melphalan, and prednisone (D-VMP) versus bortezomib, melphalan, and prednisone (VMP) in patients (pts) with transplant-ineligible newly diagnosed multiple myeloma (NDMM): ALCYONE. Presented at: 60th Annual Meeting of the American Society of Hematology. San Diego, CA; 2018. Abstract 156.

22. Facon T, Kumar SK, Plesner T, et al. Phase 3 randomized study of daratumumab plus lenalidomide and dexamethasone (D-Rd) versus lenalidomide and dexamethasone (Rd) in patients with newly diagnosed multiple myeloma (NDMM) ineligible for transplant (MAIA). Presented at: 60th Annual Meeting of the American Society of
Hematology. San Diego, CA; 2018. Abstract LBA-2.

23. Dimopoulos MA, Mateos M-V, Cavo $\mathrm{M}$, et al. One-year update of a phase 3 randomized study of daratumumab plus bortezomib, melphalan, and prednisone (D-VMP) versus bortezomib, melphalan, and prednisone (VMP) in patients (pts) with transplant-ineligible newly diagnosed multiple myeloma (NDMM): ALCYONE. Presented at: 60th Annual Meeting of the American Society of Hematology. San Diego, CA; 2018. Abstract 156.

24. Wu W, Merriman K, Nabaah A, et al. The association of diabetes and antidiabetic medications with clinical outcomes in multiple myeloma. $\mathrm{Br} \mathrm{J}$ Cancer. 2014; 111(3):628-636.

25. Bringhen $\mathrm{S}$, Mateos MV, Zweegman S, Larocca A, Falcone AP, Oriol A, et al. Age and organ damage correlate with poor survival in myeloma patients: meta-analysis of 1435 individual patient data from 4 randomized trials. Haematologica 2013;98:980-7.

26. Palumbo A, Bringhen S, Mateos M-V, Larocca A, Facon $\mathrm{T}$ et al. Geriatric assessment predicts survival and toxicities in elderly myeloma patient: an International Myeloma Working Group report. Blood 125 (13) (2015) 20682074

27. Gavriatopoulou M, Fotiou D, Roussou $\mathrm{M}$, at al. Vulnerability variables among octagenarians myeloma patients: a single-center analysis in 110 patients. ASH 2017, Blood (2017) 3152

28. Engelhardt M, Domm AS, Dold SM, Ihorst $G$ et al. A concise revised Myeloma Comorbidity Index as a valid prognostic instrument in a large cohort of 801 multiple myeloma patients. Haematologica 102 (5) (2017) 910-921

29. Murillo A, Cronin AM, Laubach JP, Hshiesh TT et al. Performance of the International Myeloma Working Group myeloma frailty score among 75 and older. J Geriatr.Oncol. 10(3) (2019) 486-489.

30. Gay F, Larocca A, Wijermans P, Cavallo F, Rossi D et al. Complete response correlates with long-term progression-free survival in elderly myeloma treated with novel agents: analysis of 1175 patients. Blood. 2011;117(11):3025-31.

31. Facon T, Mary JY, Hulin C, Benboubker L Attal M et al. Melphalan and prednisone plus thalidomide versus melphalan and prednisone alone or reduced intensity autologous stem cell transplantation in elderly patients with multiple myeloma (IFM 99-06): a randomized trial. Lancet. 2007;
370(9594):1209-18.

32. Waage A, Gimsing P, Fayers P, Abildgaard $\mathrm{N}$ et al. Melphalan and prednisone plus thalidomide or plascebo in elderly patients with multiple myeloma. Blood. 2010;116(9):1405-12.

33. Wijermans P, Schaafsma M, Termorshuizen F et al. Phase III study of the value of thalidomide added to melphalan plus prednisone in elderly patients with newly diagnosed multiple myeloma: the HOVON 49 study. J Clin Oncol. 2010;28(19):3160-6

34. Hulin C, Gacon T, Rodon P, Pegourie B et al. Efficacy of melphalan and prednisone plus thalidomide in patients older than 75 years with newly diagnosed multiple myeloma: IFM 01/01 trial. J Clin Oncol. 2009;27(22):366470.

35. Beksac M, Haznedar R, Firatli-Tuglular $\mathrm{T}$ et al. Addition of thalidomide to oral melphalan/prednisone in patients with multiple myeloma not eligible for transplantation: results of a randomized trial from the Turkish Myeloma Study Group. Eur J Haematol. 2011;86(1):1622.

36. Sacchi S, Marcheselli R, Lazzaro A, Morabito F, Fragasso a et al. A randomized trial with melphalan and prednisone plus thalidomide in newly diagnosed multiple myeloma patients not eligible for autologous stem cell transplant. Leuk Lymphoma. 2011;52(10):1942-8

37. San Miguel JF, Schlag R, Khuageva NK, Dimopoulos MA, Shpilberg O, Kropff M, et al. Bortezomib plus Melphalan and prednisone for initial treatment of multiple myeloma. N Engl J Med 2008;359:906-17.

38. Mateos M-V, Richardson PG, Schlag R, Khuageva NK, Dimopoulos MA, Shpilberg O, et al. Bortezomib plus melphalan and prednisone compared with melphalan and prednisone in previously untreated multiple myeloma: updated follow-up and im- pact of subsequent therapy in the phase III VISTA trial. J Clin Oncol 2010;28:2259-66

39. Bringhen S, Larocca A, Rossi D, Cavalli M, Genuardi M, Ria R, et al. Efficacy and safety of once-weekly bortezomib in multiple myeloma patients. Blood 2010;116:4745-53.

40. Moreau P, Pylypenko H, Grosicki S, Karamanesht I, Leleu X, Grishunina M, et al. Subcutaneous versus intravenous administration of bortezomib in patients with relapsed multiple myeloma: a randomised, phase 3, non-inferiority study. Lancet Oncol 2011;12:431-40.

41. Palumbo A, Bringhen S, Rossi D, 
Cavalli M, Larocca A, Ria R, et al. Bortezomib- melphalan-prednisonethalidomide followed by maintenance with bortezomib- thalidomide compared with bortezomib-melphalan-prednisone for initial treatment of multiple myeloma: a randomized controlled trial. J Clin Oncol 2010;28:5101-9.

42. Palumbo A, Bringhen S, Larocca A, Rossi D, Di Raimondo F, Magarotto V, et al. Bortezomib-melphalan-prednisone-thalidomide followed by maintenance with bortezomib-thalidomide compared with bortezomib-melphalanprednisone for in- itial treatment of multiple myeloma: updated follow-up and improved survival. J Clin Oncol 2014;32:634-40

43. Mateos M-V, Oriol A, Martínez-López J, Gutiérrez N, Teruel A-I, de Paz R, et al. Bortezomib, melphalan, and prednisone versus bortezomib, thalidomide, and pre- dnisone as induction therapy followed by maintenance treatment with bortezomib and thalidomide versus bortezomib and prednisone in elderly patients with un- treated multiple myelom. Lancet Oncol 2010;11:934-41

44. Mateos M-V, Oriol A, Martinez-Lopez J, Teruel A-I, Lopez de la Guia A, Lopez J, et al. GEM2005 trial update comparing VMP/VTP as induction in elderly multiple myeloma patients: do we still need alkylators? Blood 2014;124:1887-93

45. Stewart AK, Jacobus S, Fonseca R, Weiss $\mathrm{M}$ et al. Melphalan, prednisone and thalidomide versus melphalan, prednisone and lenalidomide (ECOG E1A06) in untreated multiple myeloma. Blood. 2015;126(11):1294-301.

46. Palumbo A, Hajek R, Delforge M, Kropff $\mathrm{M}$ et al. Continuous lenalidomidetratment for newly diagnosed multiple myeloma. $\mathrm{N}$ Engl $\mathrm{J}$ med. 2012;366(19):1759-69. Landmark phase III study establishing continuous lenalidomide-based therapy as a standard of care for transplant ineligible MM.

47. Benboubker L, Dimopoulos MA, Dispenzieri A, Catalano J, Belch AR, Cavo M, et al. Lenalidomide and dex- amethasone in transplant-ineligible patients with myeloma. N Engl J Med 2014;371:906-17.

48. Delforge M, Minuk L, Eisenmann J-C, Arnulf B, Canepa L, Fragasso A, et al. Health- related quality-of-life in patients with newly diagnosed multiple myeloma in the FIRST trial: lenalidomide plus low-dose dexamethasone versus melphalan, prednisone, thalidomide. Haematologica 2015;100:82633.

49. Durie BGM, Hoering A, Abidi MH, Rajkumar Sv, Epstein $\mathrm{J}$ et al. Bortezomib with lenalidomide and dexamethasone versus lenalidomide and dexamethasone alone in patients with newly diagnosed myeloma without intent for immediate autologous stemcell transplant (SWOG S0777): a randomized, open-label, phase 3 trial. Lancet. 2016. Large phase III trial establishing bortezomib-lenalidomidedexamethasone (VRd) therapy as the current standard of care for transplant ineligible MM in the US.

50. O'Donnell E, Laubach JP, Yee AJ, Huff CA et al. A phase II study of modified lenalidomide, bortezomib, and dexamethasone (RVD lite) for transplantineligicle patents with newly diagnosed multiple myeloma. Blood. 2014;124(21):3454.

51. Mateos M-V, Dimopoulos Ma Cavo M, et al. Daratumumab plus bortezomib, melphalan, and prednisone for untreated myeloma. $\mathrm{N}$ Engl J med. 2018;378(6):518-528.

52. Facon T, Kumar S, Plesner T, et al. daratumumab plus lenalidomide and dexamethasone for untreated myeloma. N Engl J Med. 2019;380(22):2104-2115.

53. Rajkumar SV. Multiple myeloma: 2020 update on diagnosis, risk-stratification and management. Am J Hematol. 2020; 95 (5):548-567.

54. Kumar SK, Callander NS, Hillengass J, Liedtke M, Baljevic M, Campagnaro E,et al: NCCN Guidelines Insights: Multiple Myeloma, version1.2020. J Natl Compr Can Netw 2019 Oct 1;17 (10):1154-1165.

55. Larocca A, Palumbo a. I how treat frag- ile myeloma patients. Blood. 2015;126(19):2179-85.

56. Kumar SK, Berdeja JG, Niesvizky R, Lional $\mathrm{S}$ et al. Safety and tolerability of ixazomib, an oral proteasome inhibitor, in combination with lenalidomide and dexamethasone in patients with previously untreated multiple myeloma: an open-label phase $1 / 2$ study. Lancet Oncol. 2014;15(13).

57. Moreau P, Masszi T, Grzasko N, et al. Oral ixazomib, lenalidomide, and dexamethasone for multiple myeloma. $\mathrm{N}$ Eng J Med. 2016;374(17):1621-34.

58. Zanwar S, Abeykoon JP, Kapoor P. Ixazomib: a novel drug for multiple myeloma. Exert Rev Hematol. 2018;11(10):761-71.

59. Lional S, Dimopoulos M, Palumbo A, et al. Elotuzumab therapy for relapsed or refractory multiple myeloma. N Eng J med. 2015;373(7):621-31.

60. Domopoulos Ma, Dytfeld D, Grosicki $\mathrm{S}$, Moreau P, et al. Elotuzumab plus pomalidomide and dexamethasone for multliple myeloma. N Engl J Med. 2018;379(19):1811-22.

61. Dimopoulos MA, Moreau P, Palumbo A, Joshua D, Pour L, et al. Carfilzomib and dexamethasone versus bortezomib and dexamethasone for patients with relapsed or refractory multiple myeloma (ENDEAVOR): a randomized, phase 3, open-label, multicenter study. Lancet Oncol. 2016; 17(1).

62. Dimopoulos MA, Stewart AK, Masszi T, Spicka L, Oriol A, et al. Carfilzomib, lenalidomide e dexamethasone in patients with relapsed multiple myeloma categorized by age: secondary analysis from the phase 3 ASPIRE study. Br J Haematol. 2017; 177(3). 404-413.

63. Dimopoulos MA, Oriol A, Nahi H, SanMiguel J, et al. Daratumumab, Lenalidomide and Dexamethasone for Multiple Myeloma. N Engl J Med 2016, 375(14), 1319-1331.

64. Palumbo A, Chanan-Khan K, Weisel K, et al. Daratumumab, Bortezomib and Dexamethasone for Multiple Myeloma. N Engl J Med 2016, 375(8) 754-766. 